\title{
Problems with time-varying extra dimensions or "Cardassian expansion" as alternatives to dark energy
}

\author{
James M. Cline \\ Theory Division, CERN, CH-1211, Geneva 23, Switzerland \\ and Physics Department, McGill University, Montréal, Québec, Canada H3A 2T8 \\ Jérémie Vinet \\ Physics Department, McGill University, Montréal, Québec, Canada H3A 2T8
}

(Received 18 November 2002; published 16 July 2003)

\begin{abstract}
It has recently been proposed that the Universe might be accelerating as a consequence of extra dimensions with time-varying size. We show that, although these scenarios can lead to acceleration, they run into serious difficulty when taking into account the limits on the time variation of the four-dimensional Newton constant. On the other hand, models of "Cardassian" expansion based on extra dimensions which have been constructed so far violate the weak energy condition for the bulk stress energy, for parameters that give an accelerating Universe.
\end{abstract}

DOI: 10.1103/PhysRevD.68.025015

PACS number(s): 11.10.Kk, 98.80.Cq, 98.80.Es

\section{INTRODUCTION}

In recent years, the physics community has witnessed a spectacular revival of interest in extra dimensions. This revival was sparked by the realization that extra dimensions need not be small or compactified to agree with current experimental and observational constraints [1-3]. The fact that they might lie within the reach of upcoming experiments while at the same time providing an elegant solution to the hierarchy problem has led to intense study of a number of models containing large and/or warped extra dimensions.

Another subject which lately has attracted much attention is the observation that the Universe is accelerating [4]. A number of hypotheses have been put forward in trying to explain this most unexpected result [5-10]. Not surprisingly, given the current interest in branes and extra dimensions, many models have appeared that make use of these very ideas to obtain an accelerating Universe [11-14].

Here, we are concerned with the mechanisms proposed in [12] and [14], where the acceleration is due to the presence of extra dimensions. In [12] the essential new ingredient is some kind of bulk stress energy which changes the form of the Friedmann equations at late times, whereas in [14] the acceleration comes from time variation of the size of the extra dimension. We examine whether these ideas can lead to acceleration while complying with well-known physical constraints, namely, on the time variation of the fourdimensional Newton constant or on the possible equation of state of the new form of stress energy.

The plan of this paper is as follows. In Sec. II, we give a brief review of the idea presented in [14]. In Sec. III, we confront this idea with experimental and observational constraints on the constancy over time of the gravitational force, showing that it runs into serious difficulty. In Sec. IV, we analyze the relationship between this model and Brans-Dicke theory. In Sec. V, we explore whether dropping the assumption that the extra dimensions are isotropic can alleviate some of the problems. In Sec. VI we show that the model that has been proposed for Cardassian acceleration violates the weak energy condition in the bulk. We discuss our results and conclude in Sec. VII.

\section{ACCELERATION FROM TIME VARIATION OF EXTRA DIMENSIONS}

The idea starts with a Universe with our usual four spacetime dimensions, supplemented by $n$ compact dimensions with time-varying size. The metric then has the form [14]

$$
\begin{aligned}
d s^{2}= & d t^{2}-a^{2}(t)\left(\frac{d r_{a}^{2}}{1-k_{a} r_{a}^{2}}+r_{a}^{2} d \Omega_{a}^{2}\right) \\
& -b^{2}(t)\left(\frac{d r_{b}^{2}}{1-k_{b} r_{b}^{2}}+r_{b}^{2} d \Omega_{b}^{2}\right),
\end{aligned}
$$

where $k_{b}=-1,0,+1$ characterizes the extra dimensions' spatial curvature. This is to be used in the action

$$
S=\int d^{4+n} x \sqrt{-\bar{g}}\left[-\frac{1}{16 \pi \bar{G}} \overline{\mathcal{R}}+\overline{\mathcal{L}}_{m}\right] .
$$

For a perfect fluid, with stress-energy tensor given by

$$
T_{N}^{M}=\operatorname{diag}\left(\bar{\rho},-\bar{p}_{a},-\bar{p}_{a},-\bar{p}_{a},-\bar{p}_{b},-\bar{p}_{b}, \ldots\right),
$$

the Einstein equations lead to

$$
\begin{aligned}
\left(\frac{\dot{a}}{a}\right)^{2}= & \frac{8 \pi \bar{G}_{-}}{3}-\frac{k_{a}}{a^{2}}-n \frac{\dot{a}}{a} \frac{\dot{b}}{b}-\frac{n(n-1)}{6}\left[\left(\frac{\dot{b}}{b}\right)^{2}+\frac{k_{b}}{b^{2}}\right], \\
\frac{\ddot{a}}{a}= & -\frac{4 \pi \bar{G}}{3}\left(\bar{\rho}+3 \bar{p}_{a}\right)-\frac{n}{2} \frac{\dot{a}}{a} \frac{\dot{b}}{b}-\frac{n(n-1)}{6}\left[\left(\frac{\dot{b}}{b}\right)^{2}\right. \\
& \left.+\frac{k_{b}}{b^{2}}\right]-\frac{n}{2} \frac{\ddot{b}}{b},
\end{aligned}
$$




$$
\begin{aligned}
\frac{\ddot{a}}{a}+\left(\frac{\dot{a}}{a}\right)^{2}= & -\frac{8 \pi \bar{G}_{\bar{p}_{b}}-\frac{k_{a}}{a^{2}}-(n-1) \frac{\dot{a}}{a} \frac{\dot{b}}{b}}{6}\left[\left(\frac{(n-1)(n-2)}{b}\right)^{2}+\frac{k_{b}}{b^{2}}\right]-\frac{(n-1)}{3} \frac{\ddot{b}}{b}
\end{aligned}
$$

Here, $\bar{G}, \bar{\rho}, \bar{p}_{a}$, and $\bar{p}_{b}$ refer to the $(4+n)$-dimensional quantities. The corresponding four-dimensional quantities are written as $G_{N}, \rho, p_{a}, p_{b}$ where $G_{N}=\bar{G} / b^{n}$ and $\left[\rho, p_{a}, p_{b}\right]=b^{n} \times\left[\bar{\rho}, \bar{p}_{a}, \bar{p}_{b}\right]$. Notice in particular the new terms in Eq. (5) that depend on the extra dimensions. We see that there is the potential for a new source of acceleration beyond those of standard cosmology. Let us now consider how constraints on the time dependence of Newton's constant constrain this possibility.

\section{CONFRONTATION WITH EXPERIMENTAL CONSTRAINTS}

It is well known that, in models with extra dimensions, the effective strength of gravity is related to the volume of the extra dimensions. This dependence can be negligible in warped geometries [2,3] but not in the type of factorizable geometry considered here. Indeed, in such a case, the effective 4D Newton constant $G_{N}$ is inversely proportional to the total volume of the extra dimensions (see, e.g., [1]). Consequently, any variation in the extra dimensions' volume will show up as a variation of $G_{N}$,

$$
G_{N} \sim b^{-n} \Rightarrow \frac{\dot{G}_{N}}{G_{N}}=-n \frac{\dot{b}}{b} .
$$

There are tight constraints on $\dot{G}_{N} / G_{N}$ from a number of experimental and observational considerations (see [15] for a thorough review). The most generous upper bounds currently give, roughly, $\left|\dot{G}_{N} / G_{N}\right|<3 \times 10^{-19} \mathrm{~s}^{-1}$. Combining this with the accepted value for the current Hubble rate [16] $\dot{a}_{0} / a_{0} \approx 2.3 \times 10^{-18} \mathrm{~s}^{-1}$ leads to

$$
\left|\frac{\dot{b}_{0}}{b_{0}}\right| \lesssim \frac{1}{10 n} \frac{\dot{a}_{0}}{a_{0}} .
$$

Given this bound, we can see immediately by looking at Eq. (5) that, in the absence of curvature in the extra dimensions, only the term involving $\ddot{b}$ is capable of providing a significant positive contribution to the acceleration. However, this term must then be of the same order as $\left(\dot{a}_{0} / a_{0}\right)^{2}$, a situation which appears somewhat unnatural given the fact that we demand that the first derivative of $b$ be much smaller. This might not be so unnatural if the extra dimensions were oscillating, since then we could accidentally be living at a time when $(\dot{b} / b)^{2} \ll \ddot{b} / b$. However, for oscillations whose period is a significant fraction of the age of the Universe, to account for acceleration that was present at a redshift of $z=1$, the effective four-dimensional theory would have to contain a nearly massless $(m \sim H)$ Brans-Dicke scalar field (the field $b$ ) with gravitational strength couplings to standard model particles. If its mass were larger than $H$, it would oscillate on time scales $m^{-1}$ much shorter than the age of the Universe. Such a light and strongly coupled particle is clearly ruled out by experimental constraints on Brans-Dicke-like theories, as we discuss in more detail below.

Let us now look at what conditions will lead to acceleration, given Eqs. (4)-(6). Using Eq. (6) to eliminate the second derivative of $b$ from Eq. (5), and Eq. (4) to eliminate $\rho$, we find that the conditions for getting a positive value of $\ddot{a} / a$ are

$$
\begin{aligned}
& \frac{\dot{b}}{b}>\frac{\dot{a}}{a}+\sqrt{\frac{(n+1)(n+2)}{n(n-1)}\left(\frac{\dot{a}}{a}\right)^{2}+2 \frac{2 n+1}{n(n-1)} \frac{k_{a}}{a^{2}}-\frac{k_{b}}{b^{2}}-\frac{16 \pi G_{N}}{n(n-1)}\left[(n-1) p_{a}-n p_{b}\right]}, \\
& \frac{\dot{b}}{b}<\frac{\dot{a}}{a}-\sqrt{\frac{(n+1)(n+2)}{n(n-1)}\left(\frac{\dot{a}}{a}\right)^{2}+2 \frac{2 n+1}{n(n-1)} \frac{k_{a}}{a^{2}}-\frac{k_{b}}{b^{2}}-\frac{16 \pi G_{N}}{n(n-1)}\left[(n-1) p_{a}-n p_{b}\right]}
\end{aligned}
$$

Comparing with the constraint (8), we can immediately rule out the first inequality (9).

Since we are concerned with acceleration in our current era of matter domination, we now set $p_{a}$ to zero. Furthermore, measurements of the cosmic microwave background (CMB) imply that our three large spatial dimensions are flat or have negligibly small curvature, so that it is appropriate to set $k_{a}=0$. Demanding that Eq. (10) not conflict with Eq. (8), we find the following:

$$
\begin{aligned}
16 \pi G_{N} p_{b}-\frac{k_{b}}{b_{0}^{2}}(n-1)< & \left(\frac{\dot{a}_{0}}{a_{0}}\right)^{2}\left[\left(1+\frac{1}{10 n}\right)^{2}(n-1)\right. \\
& \left.-\frac{(n+2)(n+1)}{n}\right]
\end{aligned}
$$

The right hand side is negative for all $n>0$, so this condition cannot be satisfied in the case studied in [14], where $k_{b}$ $=p_{b}=0$. 
Having shown that the simple flat and pressureless option is excluded, we now examine the more general cases where there can be pressure and/or curvature in the extra dimensions. If $k_{b}$ is nonzero, then it should play the role of the dark energy,

$$
-\frac{n(n-1)}{6 H^{2}} \frac{k_{b}}{b^{2}}=\Omega_{\Lambda} \sim 0.7
$$

In no case do we expect $k_{b}>0$, since this would strongly contradict the latest estimates of $\Omega_{\Lambda}$ from CMB data [17]. Therefore the inequality (11) only becomes harder to satisfy if we allow for nonzero $k_{b}$.

Since the case $p_{b}=0, k_{b}= \pm 1$ is unacceptable, suppose instead that the extra dimensions are flat, i.e., $k_{b}=0$. Then Eq. (11) requires that $p_{b}<0$. The only obvious way to do this is through the introduction of some extra matter component to the energy density which would provide negative pressure along the extra dimensions, and most likely along the ordinary dimensions as well. But this is precisely what the model was trying to avoid in the first place, by proposing the kinematics of the extra dimensions as the sole origin of the acceleration. In light of this fact, it is hard to argue that such a model supplemented with some new form of stress energy represents an improvement over other proposed explanations for dark energy.

\section{RELATION TO BRANS-DICKE THEORY}

We now analyze the relationship between the model presented above and Brans-Dicke theory [18]. Brans-Dicke (BD) gravity is a modification of general relativity where a scalar field couples to the Ricci scalar, thus yielding a theory in which the gravitational "constant" is time dependent. The action is given in the simplest case by ${ }^{1}$

$$
S=\int d^{4} x \sqrt{-g}\left(\frac{1}{16 \pi \bar{G}}\left[-\phi \mathcal{R}+\frac{\omega}{\phi} \partial_{\mu} \phi \partial^{\mu} \phi\right]+\mathcal{L}_{m}\right),
$$

where we assume that $\mathcal{L}_{m}$ refers to a perfect fluid. We generalize this slightly by adding a potential for the BD scalar:

$$
\begin{aligned}
S= & \int d^{4} x \sqrt{-g}\left(\frac{1}{16 \pi \bar{G}}\left[-\phi \mathcal{R}+\frac{\omega}{\phi} \partial_{\mu} \phi \partial^{\mu} \phi-V(\phi)\right]\right. \\
& \left.+\mathcal{L}_{m}\right) .
\end{aligned}
$$

Models of this type have been the subject of a number of papers (see, e.g., [19]). We now show explicitly the correspondence between the models obtained from Eqs. (2) and

\footnotetext{
${ }^{1}$ Note that here, as in the extra-dimensional theory, $\bar{G}$ refers to the bare gravitational coupling. The effective gravitational constant is thus $G_{N}=\bar{G} / \phi$.
}

(14). The equations of motion for BD theory with a potential and a typical Friedmann-Robertson-Walker (FRW) metric $d s^{2}=d t^{2}-a(t)^{2} d x_{i}^{2}$ are

$$
\begin{aligned}
\left(\frac{\dot{a}}{a}\right)^{2} & =\frac{8 \pi \bar{G}}{3 \phi} \rho+\frac{\omega}{6}\left(\frac{\dot{\phi}}{\phi}\right)^{2}-\frac{\dot{a}}{a} \frac{\dot{\phi}}{\phi}+\frac{V(\phi)}{6 \phi}, \\
\frac{\ddot{a}}{a}= & -\frac{4 \pi \bar{G}}{3 \phi}(\rho+3 p)-\frac{\omega}{3}\left(\frac{\dot{\phi}}{\phi}\right)^{2}-\frac{1}{2} \frac{\dot{a}}{a} \frac{\dot{\phi}}{\phi} \\
- & \frac{1}{2} \frac{\ddot{\phi}}{\phi}+\frac{V(\phi)}{6 \phi}, \\
(2 \omega+3)\left(\frac{1}{3} \frac{\dot{\phi}}{\phi}+\frac{\dot{a}}{a} \frac{\dot{\phi}}{\phi}\right) & =\frac{8 \pi \bar{G}}{3 \phi}(\rho-3 p) \\
& +\frac{1}{3}\left(2 \frac{V(\phi)}{\phi}-\frac{\partial V(\phi)}{\partial \phi}\right) .
\end{aligned}
$$

Comparing these with Eqs. (4)-(6), we see that the following choice (which was also obtained in [20]) leads to complete equivalence between both sets of equations, as long as the extra dimensional pressure term $\bar{p}_{b}$ vanishes:

$$
\begin{aligned}
\phi & \equiv b^{n}, \\
\omega & \equiv \frac{1}{n}-1, \\
V(\phi) & \equiv-n(n-1) k_{b} b^{n-2} \\
& =-n(n-1) k_{b} \phi^{1-2 / n} .
\end{aligned}
$$

We can now apply all known constraints on the parameters of BD theory to the model of [14]. We note here two important points. The first is that for a vanishing potential experimental limits demand that $\omega>1500$ [20]. Clearly, this is not possible in the present context. Furthermore, the potential given here is nontrivial for $n>2$, in which case its minimum is located at $\phi=0$, for which $G_{N}$ diverges.

What can we say about the case $\bar{p}_{b} \neq 0$ ? From the point of view of $\mathrm{BD}$ theory, we can look at this in the following way. BD theory is explicitly constructed to limit the effect of the scalar field to inducing time dependence in the strength of gravity. This means that matter is not directly affected by the presence of the scalar, so that conservation of energy is given by the standard

$$
\dot{\rho}=-3 \frac{\dot{a}}{a}(\rho+p) \Rightarrow \rho \sim a^{-3(1+\omega)}
$$

If we now look at the conservation of energy equation in the extra dimensional theory, it is given by

$$
\dot{\bar{\rho}}=-3 \frac{\dot{a}}{a}\left(\bar{\rho}+\bar{p}_{a}\right)-n \frac{\dot{b}}{b}\left(\bar{\rho}+\bar{p}_{b}\right) .
$$


Writing $\bar{p}_{a}=\omega_{a} \bar{\rho}$ and $\bar{p}_{b}=\omega_{b} \bar{\rho}$, and assuming $\omega_{a}$ and $\omega_{b}$ to be constant, we have

$$
\begin{aligned}
& \bar{\rho} \sim a^{-3\left(1+\omega_{a}\right)} b^{-n\left(1+\omega_{b}\right)} \\
\Rightarrow & \rho \sim a^{-3\left(1+\omega_{a}\right)} b^{-n \omega_{b}} .
\end{aligned}
$$

We can see that if $\bar{p}_{b}=0$ then these are reduced to the standard form (19). However, if $\bar{p}_{b} \neq 0$, conservation of energy is modified. Indeed, in order for a scalar-tensor theory to behave as the extra dimensional model does in the case where $\bar{p}_{b} \neq 0$, the energy density would need to scale as [using Eq. (18)]

$$
\begin{gathered}
\rho \sim a^{-3\left(1+\omega_{a}\right)} \phi^{-\omega_{b}} \\
\Rightarrow \dot{\rho}=-3 H\left(1+\omega_{a}\right) \rho-\omega_{b} \frac{\dot{\phi}}{\phi} \rho,
\end{gathered}
$$

leading to a theory qualitatively different from BD gravity.

\section{ANISOTROPIC EXTRA DIMENSIONS}

In the model we examined above, it was assumed that all the extra dimensions had the same scale factor. We now drop this assumption and see whether some of the difficulties we have pointed out for isotropic extra dimensions can be relieved. Such models, dubbed multidimensional cosmology, have already been extensively studied (see $[21,22]$ and references therein), but, as far as we know, not with a view toward obtaining cosmic acceleration (however, see the "Note added" at the end).

A particularly interesting aspect of such models is the fact that in the corresponding multi-scalar-tensor theory, only one scalar (corresponding to the total volume of the extra dimensions) couples to matter. This means that constraints on the time variation of Newton's constant apply only to this one field. From the point of view of the extra dimensional theory, one might therefore hope that as long as the total volume remains approximately constant, the variations of the individual dimensions can be large enough to have the desired effect on the dynamics of the Universe.

The metric for this model is given by ${ }^{2}$

$$
d s^{2}=d t^{2}-a^{2}(t) d x^{2}-\sum_{i} c_{i}^{2}(t) d \theta_{i}^{2}
$$

where $i$ runs from 1 to $n$, the number of extra dimensions. The stress-energy tensor is that of a $(4+n)$-dimensional perfect fluid:

$$
T_{n}^{m}=\operatorname{diag}\left(\bar{\rho},-\bar{p},-\bar{p},-\bar{p},-\bar{p}^{\theta_{1}}, \ldots,-\bar{p}^{\theta_{n}}\right) .
$$

The Einstein equations then lead to the following:

\footnotetext{
${ }^{2}$ Following the discussion in Sec. III, we assume from the start that the extra dimensions are flat.
}

$$
\begin{aligned}
&\left(\frac{\dot{a}}{a}\right)^{2}= \frac{8 \pi \bar{G}}{3} \bar{\rho}-\frac{\dot{a}}{a} \sum_{i} \frac{\dot{c}_{i}}{c_{i}}-\frac{1}{6}\left(\sum_{i} \frac{\dot{c}_{i}}{c_{i}}\right)^{2}+\frac{1}{6} \sum_{i}\left(\frac{\dot{c}_{i}}{c_{i}}\right)^{2}, \\
& \frac{\ddot{a}}{a}= \frac{-4 \pi \bar{G}}{3}[\bar{\rho}+3 \bar{p}]-\frac{1}{2} \frac{\dot{a}}{a} \sum_{i} \frac{\dot{c}_{i}}{c_{i}}-\frac{1}{6}\left(\sum_{i} \frac{\dot{c}_{i}}{c_{i}}\right)^{2} \\
&-\frac{1}{2} \frac{d}{d t} \sum_{i} \frac{\dot{c}_{i}}{c_{i}}-\frac{1}{3} \sum_{i}\left(\frac{\dot{c}_{i}}{c_{i}}\right)^{2}, \\
& \frac{8 \pi \bar{G}}{3} p^{\theta_{k}}-\frac{1}{3} \frac{d}{d t}\left(\frac{\dot{c}_{k}}{c_{k}}\right)-\frac{\dot{c}_{k}}{c_{k}}\left(\frac{\dot{a}}{a}+\frac{1}{3} \sum_{i} \frac{\dot{c}_{i}}{c_{i}}\right) \\
&=-\frac{4 \pi \bar{G}}{3}(\bar{\rho}-3 \bar{p})+\frac{1}{6}\left(\sum_{i} \frac{\dot{c}_{i}}{c_{i}}\right)^{2}+\frac{1}{2} \frac{\dot{a}}{a} \sum_{i} \frac{\dot{c}_{i}}{c_{i}} \\
&+\frac{1}{6} \frac{d}{d t} \sum_{i} \frac{\dot{c}_{i}}{c_{i}} .
\end{aligned}
$$

Note that there is one equation of the form (28) for each of the $n$ extra dimensions, whereas for isotropic extra dimensions, these equations were degenerate.

The same reasoning we employed in the previous section allows us to state that

$$
\left|\sum_{i} \frac{\dot{c}_{i}}{c_{i}}\right| \lesssim \frac{1}{10} \frac{\dot{a}_{0}}{a_{0}} .
$$

Notice, however, that due to the independence of the $\dot{c}_{i} / c_{i}$ 's, this in no way constrains the $\Sigma_{i}\left(\dot{c}_{i} / c_{i}\right)^{2}$ terms appearing in the Friedmann equations. This means that it is in principle possible that the sum of the squares term in Eq. (26) accounts for the missing energy density, while the $(d / d t) \sum_{i}\left(\dot{c}_{i} / c_{i}\right)$ term in Eq. (27) accounts for the acceleration. As in the isotropic case though, it seems unnatural to ask that the time derivative of a small quantity be large. Furthermore, we have not yet taken Eq. (28) into account.

Adding the $n$ equations (28) leads to

$$
\begin{aligned}
\frac{8 \pi \bar{G}}{3} \sum_{i} \bar{p}^{\theta_{i}}-\frac{1}{3} \frac{d}{d t} \sum_{i}\left(\frac{\dot{c}_{i}}{c_{i}}\right)-\sum_{i} \frac{\dot{c}_{i}}{c_{i}}\left(\frac{\dot{a}}{a}+\frac{1}{3} \sum_{i} \frac{\dot{c}_{i}}{c_{i}}\right) \\
=-n \frac{4 \pi \bar{G}}{3}(\bar{\rho}-3 \bar{p})+\frac{n}{6}\left(\sum_{i} \frac{\dot{c}_{i}}{c_{i}}\right)^{2}+\frac{n}{2} \frac{\dot{a}}{a} \sum_{i} \frac{\dot{c}_{i}}{c_{i}} \\
+\frac{n}{6} \frac{d}{d t} \sum_{i} \frac{\dot{c}_{i}}{c_{i}},
\end{aligned}
$$

which allows us to write

$$
\begin{aligned}
\frac{\ddot{a}}{a}= & -4 \pi \bar{G}\left[\frac{\bar{\rho}}{3}\left(1+\frac{3 n}{2+n}\right)+\bar{p}\left(1-\frac{3 n}{2+n}\right)+\frac{2}{2+n} \sum_{i} \bar{p}^{\theta_{i}}\right] \\
& +\frac{\dot{a}}{a} \sum_{i} \frac{\dot{c}_{i}}{c_{i}}+\frac{1}{3}\left(\sum_{i} \frac{\dot{c}_{i}}{c_{i}}\right)^{2}-\frac{1}{3} \sum_{i}\left(\frac{\dot{c}_{i}}{c_{i}}\right)^{2} .
\end{aligned}
$$


Using Eq. (29) and the fact that $\bar{p}=0$ in our current matterdominated epoch, we see that in order to obtain a positive value for $\ddot{a} / a$, we must have $\Sigma_{i} \bar{p} \theta_{i}<0$, as was the case for isotropic extra dimensions.

\section{VI. "CARDASSIAN" EXPANSION FROM EXTRA DIMENSIONS}

In the previous sections we considered time-varying extra dimensions as a source of cosmic acceleration. Also in the context of extra dimensional effects, it was recently proposed $[12,13]$ that the current era of acceleration arises from modifying the Friedmann equation by adding a term proportional to $\rho^{n}$ :

$$
H^{2}=\frac{8 \pi}{3} G\left(\rho+C \rho^{n}\right)
$$

The authors dubbed this kind of expansion "Cardassian." The new term dominates at late times if $n<1$, and if $n$ $<2 / 3$, it gives rise to a positive acceleration. This kind of behavior is qualitatively very different from the standard braneworld result which has $n=2$ [23], because it implies a modification of gravity at very low energy scales rather than very high ones. Actually, Eq. (32) is a bit misleading, in giving the impression that $\rho$ is the sole source of expansion. All of the particle physics models that have been proposed to give the modified Friedmann equation introduce an exotic new source of energy which contributes significantly to the expansion; the effects of the new matter are merely parametrized in terms of the conventional matter energy density $\rho$. In the present discussion we treat only the extra-dimensional model of Cardassian expansion on which [12] is based. (In the alternative model of [13], a new form of dark matter with an exotic confining force is hypothesized, in which the interaction energy redshifts more slowly than cold dark matter, and this gives rise to the $\rho^{\alpha}$ term. We note that there exists no realistic microscopic model for obtaining the kind of dark matter needed by [13], since it requires a new confining force with a confinement scale larger than the present horizon, and this force must be mediated by seemingly implausible objects with spatial dimensionality greater than 1.)

The Cardassian model in question is based on the work of Ref. [24], which showed that, if one parametrizes the Hubble rate in terms of the brane energy density $\rho$ (as though the bulk contribution to the energy density was hidden), then an arbitrary power $n$ can be obtained for the Friedmann equation, $H^{2} \sim \rho^{n}$. This conclusion was reached by writing down a candidate solution for the 5D Einstein equations and Israel junction conditions, and then seeing what form of the bulk stress-energy tensor would be required to make this indeed a solution to the bulk equations. Specifically, the authors consider a $5 \mathrm{D}$ metric of the form

$$
d s^{2}=e^{2 \nu(t, r)} d t^{2}-e^{2 \alpha(t, r)} d \mathbf{x}^{2}-e^{2 \beta(t, r)} d r^{2},
$$

where the extra dimension is bounded by branes at $r=0$ and at $r=l$. The metric functions are given by

$$
\beta(t, r)=\nu(t, r)=c r t^{-2 / n}, \quad \alpha(t, r)=-\frac{1}{2} \beta(t, r)+\frac{2}{3 n} \ln t,
$$

with $c=\frac{1}{3} \kappa_{5}^{2} \mu(2 / 3 n)^{2 / n}$, where $\mu$ is a dimensionful constant such that the Friedmann equation is exactly $H^{2}=(\rho / \mu)^{n}$. (No solution has been proposed that gives what one really wants, namely, a transition from $H^{2} \sim \rho$ to $H^{2} \sim \rho^{n}$.)

The size of the extra dimension is time dependent in the solution given by Eq. (34); this may be incompatible with stabilizing its size, as is usually required for a braneworld model to be compatible with fifth force constraints. However, $\beta$ asymptotically approaches a fixed value at large times, so it may be possible that such a solution is consistent with having a stabilized radion, as well as satisfying constraints on the time dependence of the gravitational force. In the present model, Newton's constant is proportional to

$$
G_{N} \propto \int_{0}^{l} e^{\beta} d r=\left.G_{N}\right|_{t \rightarrow \infty} \frac{t^{2 / n}}{c l}\left(e^{c l t^{-2 / n}}-1\right),
$$

which approaches a constant as $t \rightarrow \infty$. There seems to be sufficient freedom in the choice of parameters to ensure the relative constancy of $G_{N}$, even since the era of big bang nucleosynthesis.

However, the model runs into difficulties when we examine the equation of state of the bulk stress energy, in particular the $T_{5}^{5}$ component: we find that the weak energy condition is violated, with the pressure component $\left|T_{5}^{5}\right|$ being larger in magnitude than $T_{0}^{0}$. Specifically,

$$
\begin{aligned}
\kappa_{5}^{2} T_{0}^{0}= & \frac{e^{-2 c t^{-q} r}}{3 t^{2}}\left(q^{2}-\frac{9}{4} t^{-2 q} c^{2} r^{2} q^{2}-9 t^{-2 q+2} c^{2}\right), \\
\kappa_{5}^{2} T_{5}^{5}= & -\frac{e^{-2 c t^{-q} r}}{3 t^{2}}\left(\frac{9}{2} t^{-q} c r q-9 t^{-2 q} c^{2} r^{2} q^{2}\right. \\
& \left.-\frac{9}{4} t^{-2 q+2} c^{2}-2 q^{2}-\frac{9}{2} t^{-q} c r q^{2}+3 q\right),
\end{aligned}
$$

where $q=2 / n$. In the large-time limit, the ratio of $T_{5}^{5}$ to $T_{0}^{0}$ is

$$
\frac{T_{5}^{5}}{T_{0}{ }^{0}}=2-\frac{3}{2} n
$$

Interestingly, this quantity starts to violate the weak energy condition just for the values of $n$ where acceleration begins, namely, $n<2 / 3$. We remind the reader that such a violation implies unphysical behavior; in field theory a negative kinetic energy is implied, corresponding to a wrong-sign kinetic term which spoils unitarity.

\section{CONCLUSION}

The idea put forth in [14] that the Universe's current accelerated expansion might have purely geometrical origins 
initially sounds quite attractive. Indeed, it is easy to show that general multidimensional models can lead to our threespace accelerating without invoking new matter components with exotic equations of state. However, as we have argued, the simplest of such models are completely equivalent to a class of Brans-Dicke theories which are ruled out by experiment, either because the BD parameter $\omega$ is too small, or because the scalar field potential has a minimum for which Newton's constant diverges. The only way to make these models compatible with experimental constraints while still leading to acceleration is to invoke nonzero pressure along the extra dimensions. This, however, means that we are no longer dealing with "normal" matter, thus spoiling the model's initial motivation. This is true for anisotropic as well as for isotropic extra dimensions. From the point of view of scalar-tensor theory, we have shown that this nonvanishing pressure along the extra dimensions corresponds to modifying conservation of energy such that the theory differs from standard BD gravity.

As far as "Cardassian" expansion is concerned, we focused on a particular proposal which makes use of extra dimensions in order to obtain a modified Friedmann equation. It appears that it is possible to tune the parameters in such a way that Newton's constant tends toward a constant value fast enough to avoid any conflict with experimental evidence. However, we showed that, upon closer inspection, the bulk stress-energy tensor behaves in a way that leads to a serious difficulty. Indeed, for exactly the values of the parameter $n$ that make acceleration possible, it violates the weak energy condition. That being said, it would be very interesting if someone could construct a plausible microphysical model which leads to the Cardassian hypothesis without violating fundamental principles like causality or unitarity.
There are alternative ways [11] of using extra dimensions to explain acceleration, relying on the intrinsic curvature of branes to achieve the desired effect. Other work (e.g., $[20,25,26])$ has also investigated the cosmological implications of the evolution of the radion. Such models evade the constraints we have pointed out here, and as such are currently on sounder footing from a theoretical standpoint than the ones analyzed in this paper.

It is clear that extra dimensions lead to a number of interesting effects which might provide solutions to a number of outstanding questions in fundamental physics. In light of our results, however, it is also clear that much work needs to be done on the models we have analyzed here if they are to be considered as prime candidates in resolving the mystery of cosmological acceleration.

Note added. After completion of this work, we were informed by M. Pietroni of an anisotropic model [27] which gives a more negative equation of state $(w=-1 / 3)$ than does ordinary dark matter $(w=0)$. This result is consistent with our observation in Sec. V that negative values for the extradimensional pressure components are needed to get cosmic acceleration. The model of Ref. [27] has positive pressures, and it does not have acceleration, since $w=-1 / 3$ is the borderline value between deceleration and acceleration. We thank M. Pietroni for bringing this work to our attention. We also wish to thank U. Guenther and A. Zhuk for pointing out [22], and O. Bertolami for pointing out [9] to us.

\section{ACKNOWLEDGMENTS}

J.C. and J.V. are supported in part by grants from Canada's National Sciences and Engineering Research Council.
[1] N. Arkani-Hamed, S. Dimopoulos, and G. Dvali, Phys. Lett. B 429, 263 (1998); 436, 257 (1998); Phys. Rev. D 59, 086004 (1999).

[2] L. Randall and R. Sundrum, Phys. Rev. Lett. 83, 3370 (1999).

[3] L. Randall and R. Sundrum, Phys. Rev. Lett. 83, 4690 (1999).

[4] S. Perlmutter et al., Nature (London) 391, 51 (1998); A. Riess et al., Astron. J. 116, 1009 (1998).

[5] R.R. Caldwell, R. Dave, and P.J. Steinhardt, Phys. Rev. Lett. 80, 1582 (1998).

[6] M.S. Turner and M. White, Phys. Rev. D 56, 4439 (1997).

[7] T. Padmanabhan, Phys. Rev. D 66, 021301(R) (2002).

[8] A.Yu. Kamenshchik, U. Moschella, and V. Pasquier, Phys. Lett. B 511, 265 (2001).

[9] M.C. Bento, O. Bertolami, and A.A. Sen, Phys. Rev. D 66, 043507 (2002); 67, 063003 (2003).

[10] J.W. Moffat, astro-ph/0108201.

[11] C. Deffayet, G. Dvali, and G. Gabadadze, Phys. Rev. D 65, 044023 (2002); V. Sahni and Y. Shtanov, astro-ph/0202346; M.D. Maia, E.M. Monte, and J.M.F. Maia, astro-ph/0208223.

[12] K. Freese and M. Lewis, Phys. Lett. B 540, 1 (2002); K. Freese, hep-ph/0208264.
[13] P. Gondolo and K. Freese, hep-ph/0209322.

[14] Je-An Gu and W.-Y.P. Hwang, Phys. Rev. D 66, 024003 (2002); Je-An Gu, astro-ph/0209223.

[15] J.-P. Uzan, Rev. Mod. Phys. 75, 403 (2003).

[16] W.L. Freedman, B.F. Madore, B.K. Gibson, L. Ferrarese, D.D. Kelson, S. Sakai, J.R. Mould, R.C. Kennicutt, Jr., H.C. Ford, J.A. Graham, J.P. Huchra, S.M.G. Hughes, G.D. Illingworth, L.M. Macri, and P.B. Stetson, Astrophys. J. 553, 47 (2001).

[17] C.L. Bennett et al., astro-ph/0302207.

[18] C. Brans and R.H. Dicke, Phys. Rev. 124, 925 (1961).

[19] B.K. Sahoo and L.P. Singh, Mod. Phys. Lett. A 17, 2409 (2002); A.A. Sen, S. Sen, and S. Sehti, Phys. Rev. D 63, 107501 (2003); P. Brax and J. Martin, astro-ph/0210533; O. Bertolami and P.J. Martins, Phys. Rev. D 61, 064007 (2000); C. Santos and R. Gregory, Ann. Phys. (N.Y.) 59, 123510 (1999); J.-P. Uzan, Phys. Rev. D 59, 123510 (1999); N. Bartolo and M. Pietroni, ibid. 61, 023518 (2000); A. Liddle and R.J. Scherrer, ibid. 59, 023509 (1999).

[20] L. Perivolaropoulos and C. Sourdis, Phys. Rev. D 66, 084018 (2002).

[21] A. Zhuk, Astron. Nachr. 316, 269 (1995); U. Günther and A. 
Zhuk, Phys. Rev. D 61, 124001 (2000); Class. Quantum Grav. 15, 2025 (1998); M. Rainer and A. Zhuk, Phys. Rev. D 54, 6186 (1996); V.D. Ivashchuk and V.N. Melnikov, Int. J. Mod. Phys. D 3, 795 (1994).

[22] U. Guenther and A. Zhuk, Class. Quantum Grav. 18, 1441 (2001).

[23] P. Binetruy, C. Deffayet, and D. Langlois, Nucl. Phys. B565, 269 (2000); C. Csaki, M. Graesser, C. Kolda, and J. Terning, Phys. Lett. B 462, 34 (1999); J.M. Cline, C. Grojean, and G.
Servant, Phys. Rev. Lett. 83, 4245 (1999).

[24] D.J.H. Chung and K. Freese, Phys. Rev. D 61, 023511 (2000).

[25] Ph. Brax, C. van de Bruck, A.-C. Davis, and C.S. Rhodes, Phys. Rev. D 67, 023512 (2003); Astrophys. Space Sci. 283, 627 (2003).

[26] P. Binetruy, C. Deffayet, and D. Langlois, Nucl. Phys. B615, 219 (2001).

[27] M. Pietroni, Phys. Rev. D 67, 103523 (2003). 